Accepted by the Astrophysical Journal

Preprint typeset using LATEX style emulateapj v. 08/22/09

\title{
TWO MASSIVE, LOW-LUMINOSITY CORES TOWARD INFRARED DARK CLOUDS
}

\author{
JONATHAN J. SWIFT \\ Institute for Astronomy, 2680 Woodlawn Dr., Honolulu, HI 96822-1897 \\ Accepted by the Astrophysical Journal
}

\begin{abstract}
This article presents high-resolution interferometric mosaics in the $850 \mu \mathrm{m}$ waveband of two massive, quiescent infrared dark clouds. The two clouds were chosen based on their likelihood to represent environments preceding the formation of massive stars. The brightest compact sources detected in each cloud have masses $\approx 110 M_{\odot}$ and $\approx 60 M_{\odot}$ with radii $<0.1 \mathrm{pc}$, implying mean densities of $\langle n\rangle \approx 10^{6} \mathrm{~cm}^{-3}$ and $\langle N\rangle \approx 1 \mathrm{~g} \mathrm{~cm}^{-2}$. Supplementary data show these cores to be cold and inactive. Low upper limits to their bolometric luminosities and temperatures place them at a very early stage of evolution while current models of massive star formation suggest they have the potential to form massive stars.

Subject headings: stars: formation — ISM: clouds — ISM: structure
\end{abstract}

\section{INTRODUCTION}

It is fairly well established that low-mass stars form from the gravitational collapse of dense condensations, or cores $^{1}$, within molecular clouds (Myers \& Benson 1983 Beichman et al. 1986, Motte et al. 1998), and there are many examples of cores in nearby star-forming regions $(\lesssim 300 \mathrm{pc})$ that appear to represent the direct progenitors of low-mass stars, at least in a statistical sense (e.g., Alves et al. 2007). However, surveys of distant regions where massive stars form suffer from poor physical resolution, blending together regions with sizes comparable to clusters of stars (e.g., Shirley et al. 2003. Evans 2008). High-resolution interferometric observations can resolve these massive clumps into substructure more closely related to individual stars or stellar systems (e.g., Beuther et al. 2007; Pillai et al. 2006, Molinari et al. 2002). But in virtually all cases, these high-resolution studies have targeted regions with indications for young massive stars such as bright mid-infrared emission, masers, outflow, or compact H II.

This article presents two interferometric mosaics of infrared dark clouds (IRDCs; Simon et al. 2006a; Perault et al. 1996, Egan et al. 1998) selected to lack massive protostars yet likely to be pre-cluster clouds. Several dense cores are detected within each IRDC, but it is the most massive cores in each cloud are the focus of this article. Following a description of our observations in $\S 2$ the general properties of the IRDCs are described in $\S 3$ using new single dish data together with published data. The interferometric mosaics are then considered in $\S 4$ where the physical properties of the massive cores are presented and discussed.

\section{OBSERVATIONS AND DATA REDUCTION}

The infrared dark clouds MSXG030.88+00.13 and $M S X$ G028.53-00.25 were chosen from the catalog of

\footnotetext{
Electronic address: js@ifa.hawaii.edu

1 Following the convention presented in Williams et al. (2000), "cores" describe dense condensations of molecular gas with $M \lesssim$ $100 M_{\odot}$ that will form individual stars or small stellar systems, while "clumps" describe dense cloud structures with mass $~$ $1000 M_{\odot}$ that are more likely to form clusters of stars
}

Simon et al. (2006b) using Spitzer Galactic plane survey data (Benjamin et al. 2003; Carey et al. 2009), and SCUBA archival data (Di Francesco et al. 2008) to be massive, dense, and lack significant $24 \mu \mathrm{m}$ emission. These attributes were desired to maximize the probability that the clouds are in a state preceding the formation of massive stars. The observations are summarized in Table 1.

The target IRDCs were observed with the Submillimeter Array $^{2}$ (SMA) in its compact configuration using the $345 \mathrm{GHz}$ receivers. Mosaic observations were designed to cover the brightest SCUBA emission in each IRDC. These observations preserved the full $4 \mathrm{GHz}$ bandwidth and specified both high and low resolution correlator chunks with 0.17 and $2.8 \mathrm{~km} \mathrm{~s}^{-1}$ channel widths respectively. The data were calibrated using the MIR reduction package ${ }^{3}$. Visibility phases were corrected using quasar observations conducted in 25 minute intervals. The bandpass was calibrated with observations of the quasar $3 \mathrm{C} 279$, and Uranus was used to tie down the flux scale to an accuracy of $\sim 15 \%$. The visibility data were then output into MIRIAD ${ }^{4}$ format and inverted into the image domain. The mean rms level across the mosaic maps for $M S X \mathrm{G} 030.88+00.13$ and $M S X \mathrm{G} 028.53-00.25$ are $5.4 \mathrm{mJy}$ and $4.6 \mathrm{mJy}$ for synthesized beam sizes of $1.9^{\prime \prime} \times 1.8^{\prime \prime}$ and $2.0^{\prime \prime} \times 1.1^{\prime \prime}$ oriented at position angles of $66^{\circ}$ and $68^{\circ}$, respectively.

The James Clerk Maxwell Telescope ${ }^{5}$ (JCMT) equipped with the HARP-B receiver array and ACSIS backend (Dent et al. 2000) was used to observe our target IRDCs over several nights. Spectral lines of CO, ${ }^{13} \mathrm{CO}, \mathrm{C}^{18} \mathrm{O}, \mathrm{N}_{2} \mathrm{H}^{+}$, and $\mathrm{H}_{2} \mathrm{D}^{+}$were placed in spectral windows of $250 \mathrm{MHz}$ width and $61 \mathrm{kHz}$ resolution. Flux

\footnotetext{
2 The Submillimeter Array is a joint project between the Smithsonian Astrophysical Observatory and the Academia Sinica Institute of Astronomy and Astrophysics and is funded by the Smithsonian Institution and the Academia Sinica.

3 http://www.cfa.harvard.edu/ cqi/mircook.html

4 http://bima.astro.umd.edu/miriad

5 The James Clerk Maxwell 'Telescope is operated by The Joint Astronomy Centre on behalf of the Science and Technology Facilities Council of the United Kingdom, the Netherlands Organisation for Scientific Research, and the National Research Council of Canada.
} 
TABLE 1

Summary of OBSERVATIONS

\begin{tabular}{|c|c|c|c|c|c|}
\hline Date & Waveband & Conditions & Target $^{a}$ & Calibrators & Obs. Type \\
\hline \multicolumn{6}{|c|}{ The Submillimeter Array } \\
\hline 2007Jun24 & $350.9 \mathrm{GHz}^{\mathrm{b}}$ & $\tau_{225} \approx 0.08^{\mathrm{c}}$ & $\mathrm{B}$ & $1751+096 / 1743-038$ & 1 \\
\hline 2007Jul01 & $351.0 \mathrm{GHz}^{\mathrm{b}}$ & $\tau_{225} \approx 0.08^{\mathrm{c}}$ & B & $1751+096 / 1743-038$ & 1 \\
\hline 2007Jul07 & $350.9 \mathrm{GHz}^{\mathrm{b}}$ & $\tau_{225} \approx 0.08^{\mathrm{c}}$ & A & $1751+096 / 1743-038$ & 1 \\
\hline 2007Oct19 & $350.9 \mathrm{GHz}^{\mathrm{b}}$ & $\tau_{225} \approx 0.08^{\mathrm{c}}$ & A & $1751+096 / 1743-038$ & 1 \\
\hline 2008Jun02 & $340.5 \mathrm{GHz}^{\mathrm{b}}$ & $\tau_{225} \approx 0.15^{\mathrm{c}}$ & $\mathrm{A}, \mathrm{B}$ & $1751+096 / 1911-201$ & 1,2 \\
\hline 2008Jun15 & $340.5 \mathrm{GHz}^{\mathrm{b}}$ & $\tau_{225} \approx 0.11^{\mathrm{c}}$ & $\mathrm{A}, \mathrm{B}$ & $1751+096 / 1911-201$ & 1,2 \\
\hline \multicolumn{6}{|c|}{ James Clerk Maxwell Telescope } \\
\hline 2007Jul17 & $334.8 \mathrm{GHz} / 350.7 \mathrm{GHz}^{\mathrm{b}}$ & $\tau_{225} \approx 0.10^{\mathrm{c}}$ & $\mathrm{A}, \mathrm{B}$ & V437Sct/16293-2422/G45.1 & 3 \\
\hline 2007Jul18 & $350.7 \mathrm{GHz} / 367.3 \mathrm{GHz}^{\mathrm{b}}$ & $\tau_{225} \approx 0.06^{\mathrm{c}}$ & B & V437Sct/16293-2422/HD235858 & 3,4 \\
\hline 2007Jul19 & $334.8 \mathrm{GHz} / 350.7 \mathrm{GHz} / 367.3 \mathrm{GHz}^{\mathrm{b}}$ & $\tau_{225} \approx 0.06^{\mathrm{c}}$ & $\mathrm{A}, \mathrm{B}$ & V437Sct/16293-2422/G34.2 & 3,4 \\
\hline 2007Jul20 & $334.8 \mathrm{GHz} / 350.7 \mathrm{GHz} / 367.3 \mathrm{GHz}^{\mathrm{b}}$ & $\tau_{225} \approx 0.07^{\mathrm{c}}$ & A,B & V437Sct/16293-2422/G34.2 & 3,4 \\
\hline 2008Aug06 & $367.3 \mathrm{GHz}^{\mathrm{b}}$ & $\tau_{225} \approx 0.05^{\mathrm{c}}$ & A & WAql & 4 \\
\hline 2008Nov12 & $367.3 \mathrm{GHz}^{\mathrm{b}}$ & $\tau_{225} \approx 0.07^{\mathrm{c}}$ & A & HD179821/NMLCyg & 4 \\
\hline \multicolumn{6}{|c|}{ Canda France Hawaii Telescope } \\
\hline 2008Aug10 & $J, H, K_{s}$ & $\theta_{s} \approx 0.6^{\prime \prime d}$ & $\overline{A, B}$ & 2MASS & 5 \\
\hline 2008Aug16 & $J, H, K_{s}$ & $\theta_{s} \approx 0.7^{\prime \prime d}$ & $\mathrm{~A}, \mathrm{~B}$ & 2MASS & 5 \\
\hline
\end{tabular}

Note. - (1) Mosaic observations, (2) Targeted (single pointing) observations, (3) raster scan mapping, (4) jiggle chop observations (5) direct imaging.

a Target A corresponds to MSX G030.88+00.13, and target B corresponds to MSX G028.53-00.25.

b Local oscillator frequency.

c Atmospheric opacity at $225 \mathrm{GHz}$.

d Full width at half maximum of the point spread function.

levels are expected to be accurate to $15 \%$ and the pointing accuracy of the final maps are estimated to be better than $3.5^{\prime \prime}$. The Starlink software suite was used to grid and output the data into FITS format and IDL was used to perform final calibrations and coadding. A main beam efficiency $\eta_{\mathrm{MB}}=0.7$ is used to convert corrected antenna temperatures to main beam temperatures.

The Canada-France Hawaii Telescope ${ }^{6}$ (CFHT) was used to observe our target clouds using the WIRCam infrared detector (Puget et al. 2004). The observations were carried out in queue mode over the course of two photometric nights. The pre-processed data (de-biased, flat-fielded and sky-subtracted by the CFHT pipeline) were downloaded and further reduced using the TERAPIX $^{7}$ software suite. The images were registered, combined by weighted mean, and tied to the 2 MASS $^{8}$ point source catalog flux scale to an accuracy of better than 0.02 magnitudes.

\section{THE IRDC ENVIRONMENTS}

Figures $1 \mathrm{a}$ and $1 \mathrm{~b}$ show images from the Spitzer GLIMPSE survey in which the mid-infrared extinction features that define the clouds can be clearly seen. Contours of velocity integrated ${ }^{13} \mathrm{CO}(3-2)$ overlay the images in yellow. MSX G030.88+00.13 has 2 velocity com-

6 The Canada-France-Hawaii Telescope (CFHT) is operated by the National Research Council of Canada, the Institut National des Sciences de l'Univers of the Centre National de la Recherche Scientifique of France, and the University of Hawaii.

7 http://terapix.iap.fr

8 'The 'Two Micron All Sky Survey is a joint project of the University of Massachusetts and the Infrared Processing and Analysis Center/California Institute of Technology, funded by the National Aeronautics and Space Administration and the National Science Foundation. ponents along the line-of-sight with centroids at 95 and $107 \mathrm{~km} \mathrm{~s}^{-1}$ with respect to the local standard of rest. The higher velocity component shown in Figure $1 \mathrm{a}$ at ${ }^{13} \mathrm{CO}$ emission levels of $(5+3 n) \mathrm{K} \mathrm{km} \mathrm{s}^{-1}(n=0,1,2 \ldots)$ is widespread across the region suggesting that the IRDC is part of a larger complex including the bright infrared cluster to the northwest. MSX G028.53-00.25 has a single velocity component in ${ }^{13} \mathrm{CO}$ at $87 \mathrm{~km} \mathrm{~s}^{-1}$ shown at contour levels of $(6+2 n) \mathrm{Kkm} \mathrm{s}^{-1}$ seen to be spatially confined to the region of mid-infrared extinction. The presence of high volume density gas in these clouds is confirmed with detections of $\mathrm{N}_{2} \mathrm{H}^{+}(4-3)$ shown as magenta contours at levels of $(1+0.5 n) \mathrm{K} \mathrm{km} \mathrm{s}^{-1}$ and $(1+0.4 n) \mathrm{K} \mathrm{km} \mathrm{s}^{-1}$ for Figures $1 \mathrm{a}$ and $1 \mathrm{~b}$, respectively.

The velocity centroid of $M S X \mathrm{G} 030.88+00.13$ places it at the tangent point of Galactic rotation in this direction. Therefore a distance of $7.2 \mathrm{kpc}$ can be derived using the simple geometric relationship $d=R_{0} \cos (l) \cos (b)$, where $R_{0}$ is taken to be $8.4 \mathrm{kpc}$ (Ghez et al. 2008, Reid et al. 2009). The distance to $M S X$ G028.53-00.25 is taken to be $5.4 \mathrm{kpc}$ (Rathborne et al. 2006). The errors on these kinematic distances may be $15 \%$ or more due to noncircular motions in the Galaxy (e.g., Roman-Duval et al. 2009). The masses of the IRDCs are estimated to be several thousand solar masses based on CO isotopologue emission assuming $15 \mathrm{~K}$ gas in local thermodynamic equilibrium, SCUBA $850 \mu \mathrm{m}$ continuum emission, and virial equilibrium.

There is no $\lambda 20 \mathrm{~cm}$ or $\lambda 6 \mathrm{~cm}$ emission detected toward either IRDC (Helfand et al. 2006; White et al. 2005). A total $\lambda 20 \mathrm{~cm}$ flux of $250 \mathrm{mJy}$ seen toward the IR bright cluster in Figure 17a suggests the presence of a B0 star (Giveon et al. 2005). Two class II methanol masers are seen in the field of $M S X$ G030.88+00.13 with velocities in rough agreement with the cloud (Pestalozzi et al. 2005). 

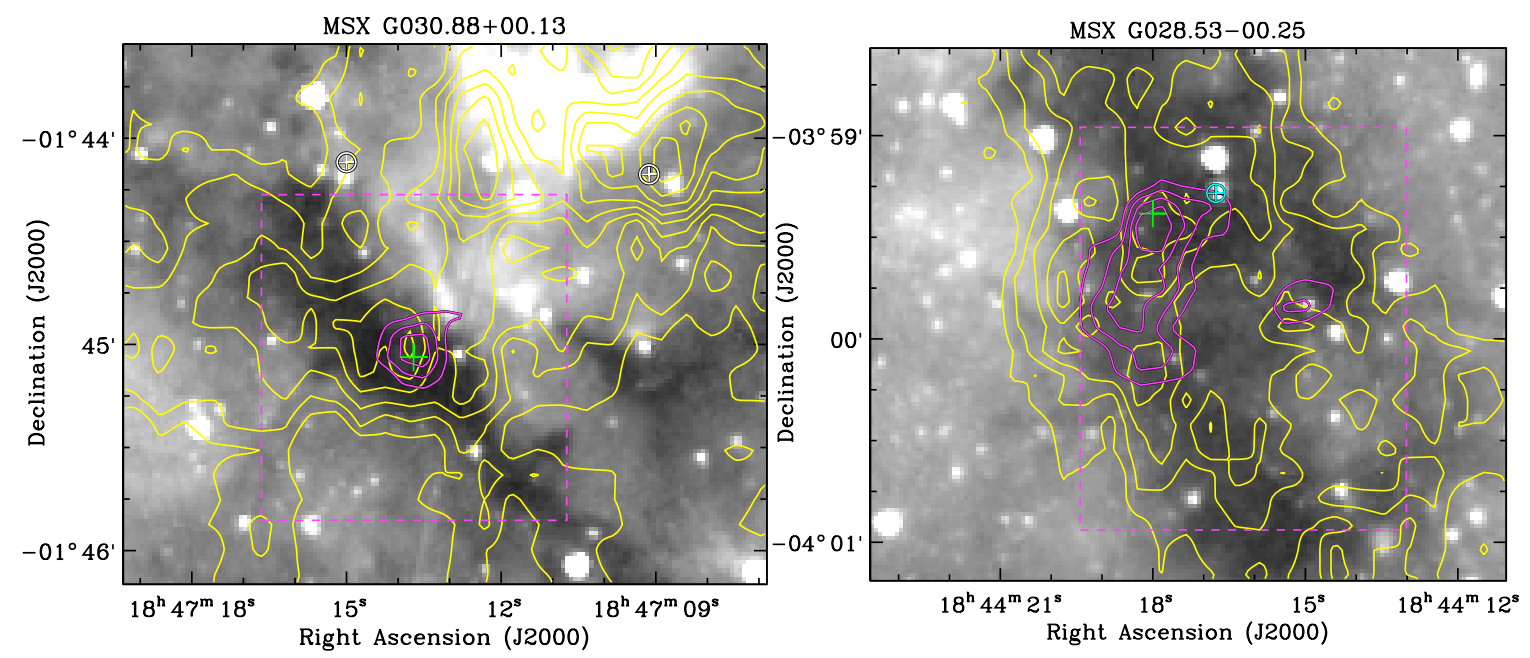

FIG. 1. - Gray scale images of $5.8+8.0 \mu \mathrm{m}$ emission toward two infrared dark clouds. Contours of ${ }^{13} \mathrm{CO}(3-2)$ emission are shown in yellow and contours of $\mathrm{N}_{2} \mathrm{H}^{+}(4-3)$ are shown in magenta. The circled crosses denote the positions of methanol (white) and water (cyan) masers. The positions of the brightest compact cores detected by the SMA (see $\S 4.1$ are shown with the green cross.

No class II masers are seen in the $M S X$ G028.53-00.25 field. However, one water maser has been detected toward an embedded source in this cloud (Wang et al. 2006). Fits to the spectral energy distributions of MIPS $24 \mu \mathrm{m}$ sources within the IRDCs suggest they are not massive stars (Robitaille et al. 2006, 2007). Although given the large column depths observed toward the IRDCs and their distances, it is possible that more protostars are embedded in the cloud than are detected in the Spitzer data. We estimate that less than $\sim 100$ stars are embedded within the IRDC based on extrapolations from mid-infrared emission from nearby, intermediatemass star forming regions (Padgett et al. 2008, Rebull et al. 2007) and a Salpeter stellar mass function with a lognormal turnover (Salpeter 1955, Chabrier 2003).

\section{HIGH-RESOLUTION SUB-MILLIMETER WAVE IMAGING}

Figures $2 \mathrm{a}$ and $2 \mathrm{~b}$ show the contours of the SMA mosaics overlaid on Spitzer mid-infrared composite images. The brightest sources in each mosaic dominate the compact sub-millimeter wave emission toward the IRDCs. These massive cores lie near the near the peak of the SCUBA emission and are isolated with respect to other compact sources. They are also isolated in mass with the next most massive cores in each cloud being more than a factor of 3 less massive. Table 2 displays the physical characteristics of the two compact submillimeter sources.

\subsection{Two Massive, Low-Luminosity Cores}

The derived masses of the two cores are $\approx 110 M_{\odot}$ and $\approx 60 M_{\odot}$ with densities of $\langle n\rangle \approx 10^{6} \mathrm{~cm}^{-3}$ and $\langle N\rangle \approx 1 \mathrm{~g} \mathrm{~cm}^{-2}$. Their free fall, or dynamical timescales are thus a few $10^{4}$ yrs implying that they are most likely short lived structures. Both cores appear marginally resolved, but are detected in the longest baseline data suggesting that perhaps there are resolved and unresolved components.

Figure 3 shows the spectral energy distributions of the cores consisting of upper limits from $1.25 \mu \mathrm{m}$ to $70 \mu \mathrm{m}$ and $853 \mu \mathrm{m}$ fluxes from Table 2. A best fit gray body curve with $\tau=\left(\nu / \nu_{\mathrm{c}}\right)^{2}$, and $\nu_{\mathrm{c}}=6 \mathrm{THz}($ WardThompson et al. 2002 limits the envelope temperatures while summation under the solid curves of Figure 3 provide upper limits to the bolometric luminosities and temperatures (Myers \& Ladd 1993).

High values of $M_{\mathrm{env}} / L_{\mathrm{bol}}^{0.6} \approx 2.8$ in solar units and low values for $T_{\mathrm{bol}} \lesssim 30 \mathrm{~K}$ for these cores are signs of extreme youth. Class 0 protostars, the earliest SED class, are marked by $M_{\text {env }} / L_{\text {bol }}^{0.6}>0.4$ Bontemps et al. 1996, Andre et al. 2000) and $T_{\text {bol }} \lesssim 70 \mathrm{~K}$ (Chen et al. 1995). Given the large distances to these cores and the photometric sensitivity, the existence of a low luminosity sources deeply embedded within the cores cannot be ruled out. However, it is clear that no massive protostars exist in these cores.

Across the SMA bandpass, the cores are only detected in $\mathrm{CO}(3-2)$ and $\mathrm{HCO}^{+}(4-3)$. The $\mathrm{CO}(3-2)$ is strongly filtered by the interferometer making a useful interpretation of the emission from this low density gas tracer difficult. However, no clear indication of outflow is seen toward either core in the single dish or interferometric data further indicating their extreme youth. Upper limits to $\mathrm{SO}\left(8_{9}-7_{8}\right)$ and $\mathrm{CH}_{3} \mathrm{OH}\left(13_{7,7}-12_{7,6}\right)$ emission highlight the difference between these cores and the spectral signatures of hot cores such as Orion KL (Schilke et al. 1997).

Figure 4 shows the composite spectra of molecular transitions tracing dense gas toward the two cores with the velocity scale shifted relative to the centroid velocity of $\mathrm{N}_{2} \mathrm{H}^{+}(4-3)$ emission measured to be $107.2 \mathrm{~km} \mathrm{~s}^{-1}$ and $86.8 \mathrm{~km} \mathrm{~s}^{-1}$ for core 1 and 2, respectively. Core 1 shows an $\mathrm{HCO}^{+}$emission feature that lies blueward of the systemic velocity. This may be an indication of $\mathrm{HCO}^{+}$selfabsorption, but there is also the possibility that the emission in the central channels is widespread and filtered out by the interferometer. Core 2 shows a weak feature in the $\mathrm{HCO}^{+}$spectral window blueward of its systemic velocity that may be a sign of a similar spectral feature to core 1.

Also shown in Figure 4 are spectra of ortho$\mathrm{H}_{2} \mathrm{D}^{+}\left(1_{1,0}-1_{1,1}\right)$ toward the cores. Core 1 shows an emission feature with $4.3 \sigma$ significance centered at 

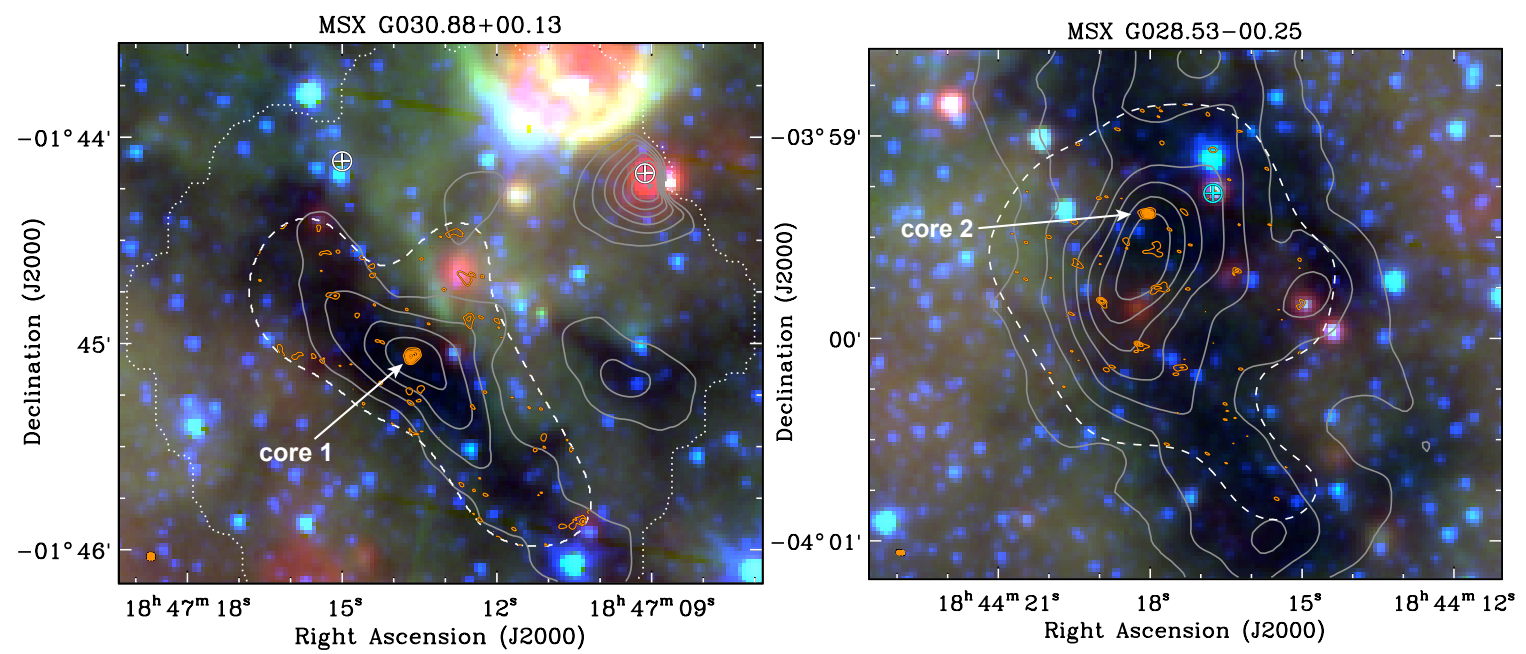

FIG. 2. - Spitzer composite images (red = MIPS $24 \mu \mathrm{m}$, green $=$ IRAC $8.0 \mu \mathrm{m}+5.8 \mu \mathrm{m}$, blue $=$ IRAC $4.5 \mu \mathrm{m}+3.6 \mu \mathrm{m}$ ) overlaid with contours of sub-millimeter continuum emission from the SMA mosaic data at levels of $3 \sigma n$ (orange) and SCUBA archival data shown at levels of $250 n$ mJy (gray) with $n=1,2,3, \ldots$ The coverage of the SMA and SCUBA data are outlined with a dashed and dotted contour, respectively.

TABLE 2

Massive, Low-Luminosity Cores

\begin{tabular}{cccccccccccc}
\hline \hline Name & $\begin{array}{c}\text { R.A. } \\
(J 2000)\end{array}$ & $\begin{array}{c}\text { Decl. } \\
(J 2000)\end{array}$ & $\begin{array}{c}F_{\text {peak }} \\
(\mathrm{mJy} / \mathrm{bm})\end{array}$ & $\begin{array}{c}F_{\text {int }} \\
(\mathrm{mJy})\end{array}$ & $\begin{array}{c}\mathrm{Mass}^{\mathrm{a}} \\
\left(M_{\odot}\right)\end{array}$ & $\begin{array}{c}r^{\mathrm{b}} \\
(\mathrm{pc})\end{array}$ & $\begin{array}{c}\langle n\rangle \\
\left(10^{6} \mathrm{~cm}^{-3}\right)\end{array}$ & $\begin{array}{c}\langle N\rangle \\
\left(\mathrm{g} \mathrm{cm}^{-2}\right)\end{array}$ & $\begin{array}{c}T_{\text {env }} \\
(\mathrm{K})\end{array}$ & $\begin{array}{c}T_{\text {bol }} \\
(\mathrm{K})\end{array}$ & $\begin{array}{c}L_{\text {bol }} \\
\left(L_{\odot}\right)\end{array}$ \\
\hline Core 1 & $18: 47: 13.7$ & $-01: 45: 03.7$ & 119 & 255 & 110 & 0.082 & 0.82 & 1.1 & $<19$ & $<31$ & $<460$ \\
Core 2 & $18: 44: 18.0$ & $-03: 59: 23.0$ & 118 & 224 & 60 & 0.056 & 1.5 & 1.3 & $<18$ & $<30$ & $<170$ \\
\hline
\end{tabular}

a Masses estimated using a dust temperature of $15 \mathrm{~K}$ and an opacity $\kappa=0.019 \mathrm{~cm}^{2} \mathrm{~g}^{-1}$ derived from (Ossenkopf \& Henning 1994 Table 1).

b Radii are derived by deconvolving the synthesized beam from the effective radius, $r=\sqrt{R_{\text {eff }}^{2}-R_{\text {beam }}^{2}}$, where $R_{\text {eff }}=\sqrt{A / \pi}$ and $A$ is the area contained within a $3 \sigma$ contour.

$106.6 \mathrm{~km} \mathrm{~s}^{-1}$ with a full width of $0.9 \pm 0.3 \mathrm{~km} \mathrm{~s}^{-1}$. Core 2 shows a broader feature with $2.0 \pm 0.7 \mathrm{~km} \mathrm{~s}^{-1}$ width at a $3.1 \sigma$ significance level. This molecular species is thought to trace cold, dense, and chemically evolved gas in the deep interior of pre- or proto-stellar cores (Walmsley et al. 2004, Vastel et al. 2006, Caselli et al.|2008).

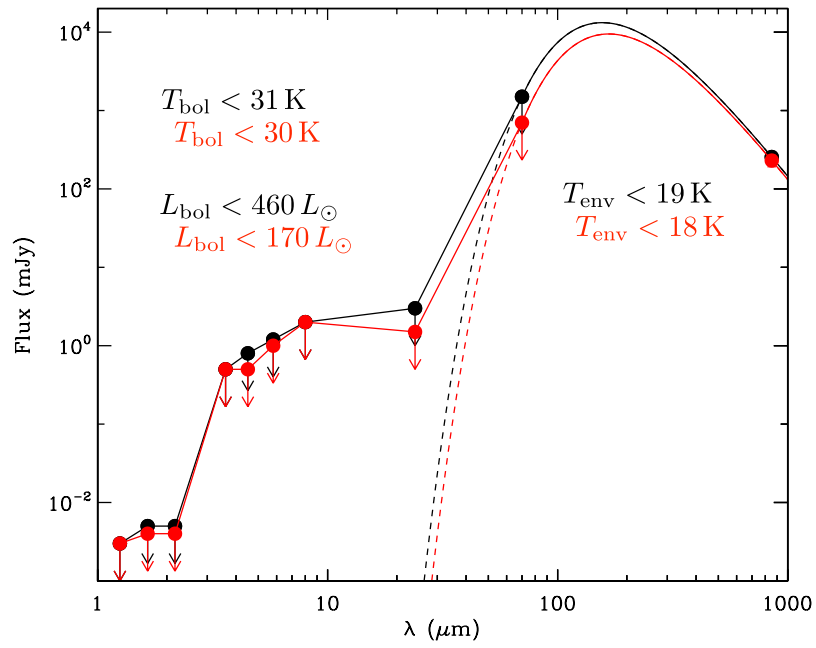

FIG. 3. - Spectral energy distributions of core 1 (black) and core 2 (red). The detections at $853 \mu \mathrm{m}$ have error bars smaller than the symbol size, while upper limits at wavelengths from 1.25 to $70 \mu \mathrm{m}$ are shown
Following Caselli et al. (2008), the integrated line fluxes of $0.13 \mathrm{~K} \mathrm{~km} \mathrm{~s}^{-1}$ and $0.17 \mathrm{~K} \mathrm{~km} \mathrm{~s}^{-1}$ for core 1 and 2 respectively translate to column densities of $N\left(\mathrm{H}_{2} \mathrm{D}^{+}\right) \approx$ $10^{11} \mathrm{~cm}^{-2}$ using a kinetic temperature $T_{\mathrm{k}}=15 \mathrm{~K}$ and a critical density $n_{\mathrm{cr}}=10^{6} \mathrm{~cm}^{-3}$. The fractional abundances of ortho- $\mathrm{H}_{2} \mathrm{D}^{+}$are $N\left(\mathrm{H}_{2} \mathrm{D}^{+}\right) / N\left(\mathrm{H}_{2}\right) \approx 3-5 \times$ $10^{-13}$. Varying $T_{\mathrm{k}}$ from 10 to $15 \mathrm{~K}$ and $N_{\mathrm{cr}}$ from $10^{5}$ to $10^{6} \mathrm{~cm}^{-3}$ changes these values by a factor of about 2 . These numbers are significantly smaller than the values found for low-mass pre- and proto-stellar cores (Vastel et al. 2006, Caselli et al. 2008) as well as in Orion B (Harju et al. 2006). This could be due to an intrinsic dearth of $\mathrm{H}_{2} \mathrm{D}^{+}$. However, given the poor physical resolution of our single dish observations and the propensity for $\mathrm{H}_{2} \mathrm{D}^{+}$to trace the innermost regions of cores, it may be that this discrepancy is due in large part to beam dilution.

\subsection{Discussion}

The signposts of massive star formation toward $M S X \mathrm{G} 030.88+00.13$ lend credence to the idea that there might exist regions within the cloud where massive stars will, but have not yet, formed. The characteristics of core 1 make it a good candidate for one such region. While MSXG028.53-00.25 currently shows no signs of high-mass star formation, core 2 shares similar traits to core 1 as being a potential massive star precursor.

The bolometric luminosity of cores versus the enve- 


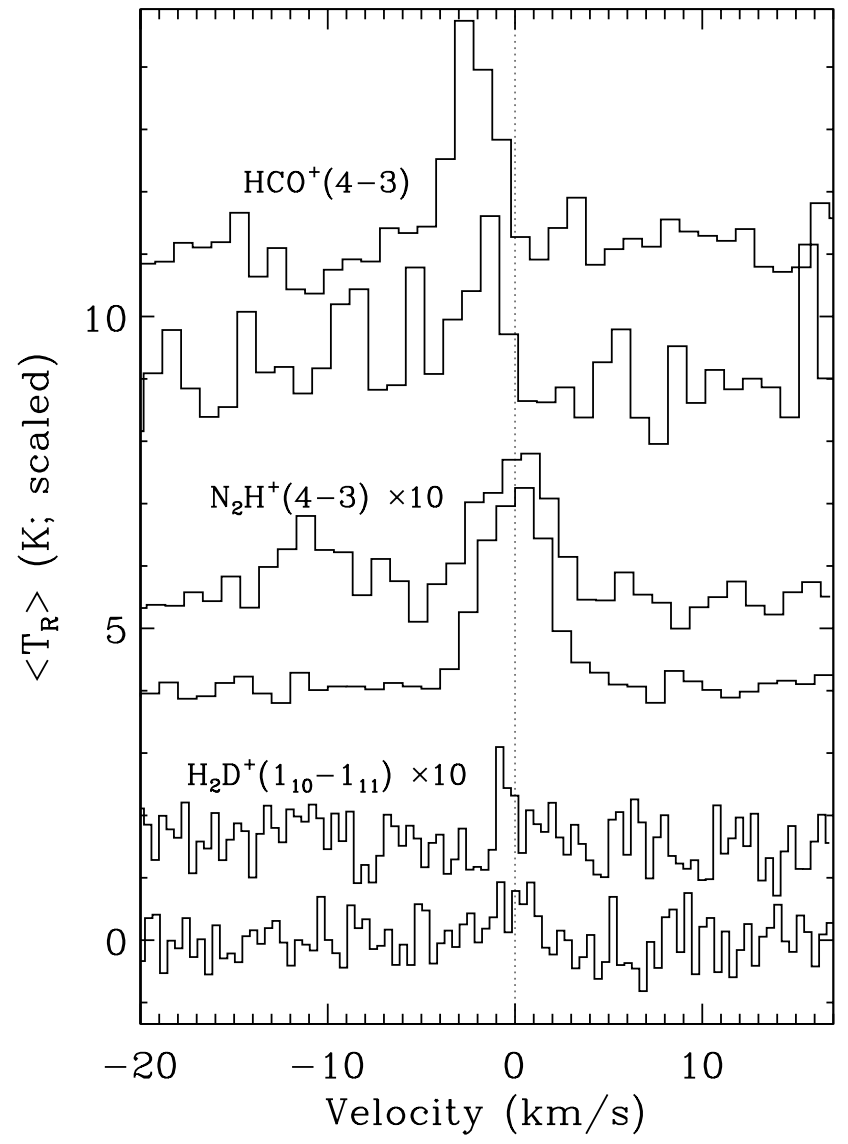

FIG. 4.- Average spectra of high-dipole moment molecules toward core 1 (top) and core 2 (bottom) in molecular transitions tracing high volume density gas. The $\mathrm{N}_{2} \mathrm{H}^{+}$and $\mathrm{H}_{2} \mathrm{D}^{+}$spectra are scaled by a factor of 10 for the plot. Velocities are with respect to the centroid of $\mathrm{N}_{2} \mathrm{H}^{+}$emission for each core measured from a Gaussian fit to the line profiles.

lope mass, or the $L_{\mathrm{bol}}-M_{\mathrm{env}}$ diagram is a useful parameter space to visualize the evolution of cores into stars (e.g., Bontemps et al. 1996). Figure 5 shows data from several previous studies of massive star formation plotted on the $L_{\mathrm{bol}}-M_{\mathrm{env}}$ diagram (see figure caption). The linear trend in log-log space is typical for such studies. Evolutionary tracks based on the turbulent core model of McKee \& Tan (2003) calibrated by the data of Molinari et al. (2008) are also overlaid on the plot. The positions of core 1 and 2 from this study are seen to lie well below the trend set by previous data, another indication that they are at an early evolutionary stage. According to the Molinari et al. (2008) evolutionary tracks, core 1 is on track to evolve into a star with $L_{\text {bol }} \approx 10^{4} L_{\odot}$, or of an early B spectral type while core 2 is set to evolve into a star with $L_{\text {bol }} \approx 2 \times 10^{3} L_{\odot}$, or a mid-B spectral class.

The final stellar masses implied from the evolutionary tracks in Figure 5 may be underestimates. The star formation efficiency of dense cores is expected to be between 25-50\% (Matzner \& McKee 2000, Alves et al. 2007). Therefore if these cores collapse monolithically they have sufficient mass to form stars with mass between 15 and $60 M_{\odot}$. Indeed, the evolutionary models of
André et al. (2008) put core 1 on track to evolve into a star of $\approx 50 M_{\odot}$ and core 2 into a star with $\approx 25 M_{\odot}$. However, it is not possible to tell from our data whether these cores will undergo monolithic collapse or will further fragment into smaller cores. Observations resolving the expected fragmentation length scales $(\sim 0.03 \mathrm{pc})$ are needed.

The large millimeter continuum survey of the Cygnus $\mathrm{X}$ region has also revealed dense and massive cores at an early stage of evolution (Motte et al. 2007). However, of the most massive cores in their sample $\left(M \geq 40 M_{\odot}\right)$, $61 \%$ have $21 \mu \mathrm{m} M S X$ sources associated with them, and the remaining either contain compact $\mathrm{HII}$, show $70 \mu \mathrm{m}$ emission above our scaled detection limits (S. Bontemps, F. Motte, private communication), or have $\mathrm{SiO}$ line strengths and widths that indicate protostellar activity.

Therefore it seems that the cores of this study are unique, and their existence may have implications for our understanding of massive star formation. The selection criteria and use of high-resolution in the sub-millimeter wave band are key components to these discoveries. As the characterizations of IRDCs progress (e.g., Chambers et al. 2009) further high-resolution studies of pre-cluster environments will provide statistics on the frequency and nature of these kinds of objects.

\section{SUMMARY}

This article presents interferometric mosaic observations in the $850 \mu \mathrm{m}$ waveband toward two infrared dark clouds chosen to be likely representations of pre-cluster environments in the Galaxy. Both clouds are massive and dense but show no sign of massive star formation. The most massive cores in each mosaic dominate the compact $850 \mu \mathrm{m}$ emission and are spatially isolated near the peak of the low-resolution continuum emission. With masses of 110 and $60 M_{\odot}$ average densities of $\langle n\rangle \approx 10^{6} \mathrm{~cm}^{-3}$ and $\langle N\rangle \approx 1 \mathrm{~g} \mathrm{~cm}^{-2}$ they have the potential to form massive stars. However, upper limits to their bolometric temperatures and luminosities, no clear indication of $\mathrm{CO}$ outflow, and their relatively featureless spectra all indicate a very early stage of evolution. Detections of ortho- $\mathrm{H}_{2} \mathrm{D}^{+}\left(1_{1,0}-1_{1,1}\right)$ and $\mathrm{N}_{2} \mathrm{H}^{+}(4-3)$ support the interpretation that these cores are in a cold and dense state preceding significant star formation activity. The comparison of these data with theoretical and observational studies of massive star forming regions suggest that these cores occupy a unique region of $L_{\mathrm{bol}}-M_{\mathrm{env}}$ parameter space placing them on track to evolve into stars anywhere from mid to early B stars up to $\mathrm{O}$ stars.

The author is grateful for the referee's suggestions as well as the helpful input provided by many people including David Jewitt, John Johnson, Sylvain Bontemps, Frederique Motte, Jonathan Williams, Emeric Le Floc'h, Qizhou Zhang, Steve Longmore, Thushara Pillai and Jill Rathborne.

\section{REFERENCES}




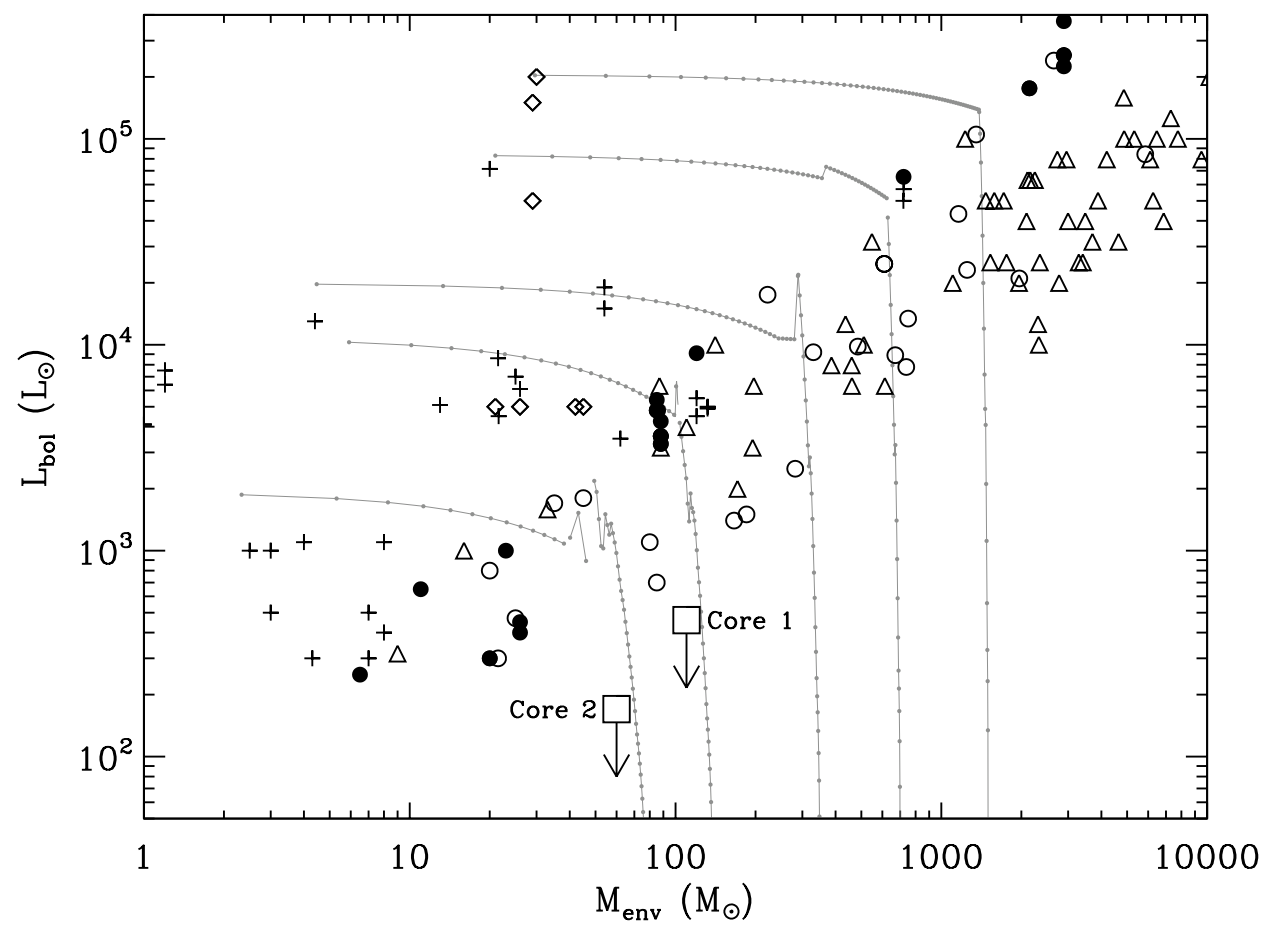

FIG. 5. $-L_{\text {bol }}-M_{\text {env }}$ diagram displaying published data for massive star forming cores [open triangles (Sridharan et al. 2002 Beuther et al. 2002 ); diamonds (André et al. 2008); open circles, filled circles and crosses (identical designations as in Molinari et al. 2008)]. Evolutionary tracks are from Molinari et al. (2008) marked in time steps of $10^{4}$ years for the vertical branch and $10^{5}$ years for the horizontal branch. The positions of core 1 and 2 are shown as upper limits in the diagram.

Andre, P., Ward-Thompson, D., \& Barsony, M. 2000, Protostars and Planets IV, 59

Beichman, C. A., Myers, P. C., Emerson, J. P., Harris, S., Mathieu, R., Benson, P. J., \& Jennings, R. E. 1986, ApJ, 307, 337

Benjamin, R. A., et al. 2003, PASP, 115, 953

Beuther, H., Leurini, S., Schilke, P., Wyrowski, F., Menten, K. M., \& Zhang, Q. 2007, A\&A, 466, 1065

Beuther, H., Schilke, P., Menten, K. M., Motte, F., Sridharan, T. K., \& Wyrowski, F. 2002, ApJ, 566, 945

Bontemps, S., Andre, P., Terebey, S., \& Cabrit, S. 1996, A\&A, 311,858

Carey, S. J., et al. 2009, PASP, 121, 76

Caselli, P., Vastel, C., Ceccarelli, C., van der Tak, F. F. S., Crapsi, A., \& Bacmann, A. 2008, A\&A, 492, 703

Chabrier, G. 2003, PASP, 115, 763

Chambers, E. T., Jackson, J. M., Rathborne, J. M., \& Simon, R. 2009, ApJS, 181, 360

Chen, H., Myers, P. C., Ladd, E. F., \& Wood, D. O. S. 1995, ApJ, 445, 377

Dent, W., et al. 2000, in Astronomical Society of the Pacific Conference Series, Vol. 217, Imaging at Radio through Submillimeter Wavelengths, ed. J. G. Mangum \& S. J. E. Radford, 33-+

Di Francesco, J., Johnstone, D., Kirk, H., MacKenzie, T., \& Ledwosinska, E. 2008, ApJS, 175, 277

Egan, M. P., Shipman, R. F., Price, S. D., Carey, S. J., Clark, F. O., \& Cohen, M. 1998, ApJ, 494, L199+

Evans, II, N. J. 2008, in Astronomical Society of the Pacific Conference Series, Vol. 390, Pathways Through an Eclectic Universe, ed. J. H. Knapen, T. J. Mahoney, \& A. Vazdekis, $52-+$

Ghez, A. M., et al. 2008, ApJ, 689, 1044

Giveon, U., Becker, R. H., Helfand, D. J., \& White, R. L. 2005, AJ, 130,156

Harju, J., et al. 2006, A\&A, 454, L55

Helfand, D. J., Becker, R. H., White, R. L., Fallon, A., \& Tuttle, S. 2006, AJ, 131, 2525

Matzner, C. D., \& McKee, C. F. 2000, ApJ, 545, 364

McKee, C. F., \& Tan, J. C. 2003, ApJ, 585, 850

Molinari, S., Pezzuto, S., Cesaroni, R., Brand, J., Faustini, F., \& Testi, L. 2008, A\&A, 481, 345

Molinari, S., Testi, L., Rodríguez, L. F., \& Zhang, Q. 2002, ApJ, 570,758

Motte, F., Andre, P., \& Neri, R. 1998, A\&A, 336, 150
Motte, F., Bontemps, S., Schilke, P., Schneider, N., Menten, K. M., \& Broguière, D. 2007, A\&A, 476, 1243

Myers, P. C., \& Benson, P. J. 1983, ApJ, 266, 309

Myers, P. C., \& Ladd, E. F. 1993, ApJ, 413, L47

Ossenkopf, V., \& Henning, T. 1994, A\&A, 291, 943

Padgett, D. L., et al. 2008, ApJ, 672, 1013

Perault, M., et al. 1996, A\&A, 315, L165

Pestalozzi, M. R., Minier, V., \& Booth, R. S. 2005, A\&A, 432, 737

Pillai, T., Wyrowski, F., Menten, K. M., \& Krügel, E. 2006, A\&A, 447, 929

Puget, P., et al. 2004, in Society of Photo-Optical

Instrumentation Engineers (SPIE) Conference Series, Vol. 5492, Society of Photo-Optical Instrumentation Engineers (SPIE) Conference Series, ed. A. F. M. Moorwood \& M. Iye, 978-987

Rathborne, J. M., Jackson, J. M., \& Simon, R. 2006, ApJ, 641, 389

Rebull, L. M., et al. 2007, ApJS, 171, 447

Reid, M. J., et al. 2009, ArXiv e-prints

Robitaille, T. P., Whitney, B. A., Indebetouw, R., \& Wood, K. 2007, ApJS, 169, 328

Robitaille, T. P., Whitney, B. A., Indebetouw, R., Wood, K., \& Denzmore, P. 2006, ApJS, 167, 256

Roman-Duval, J., Jackson, J. M., Heyer, M., Johnson, A.

Rathborne, J., Shah, R., \& Simon, R. 2009, ApJ, 699, 1153

Salpeter, E. E. 1955, ApJ, 121, 161

Schilke, P., Groesbeck, T. D., Blake, G. A., \& Phillips, T. G. 1997, ApJS, 108, 301

Shirley, Y. L., Evans, II, N. J., Young, K. E., Knez, C., \& Jaffe, D. T. 2003, ApJS, 149, 375

Simon, R., Jackson, J. M., Rathborne, J. M., \& Chambers, E. T. 2006a, ApJ, 639, 227

Simon, R., Rathborne, J. M., Shah, R. Y., Jackson, J. M., \& Chambers, E. T. 2006b, ApJ, 653, 1325

Sridharan, T. K., Beuther, H., Schilke, P., Menten, K. M., \& Wyrowski, F. 2002, ApJ, 566, 931

Vastel, C., Caselli, P., Ceccarelli, C., Phillips, T., Wiedner, M. C., Peng, R., Houde, M., \& Dominik, C. 2006, ApJ, 645, 1198

Walmsley, C. M., Flower, D. R., \& Pineau des Forêts, G. 2004 A\&A, 418, 1035

Wang, Y., Zhang, Q., Rathborne, J. M., Jackson, J., \& Wu, Y. 2006, ApJ, 651, L125

Ward-Thompson, D., André, P., \& Kirk, J. M. 2002, MNRAS, 329,257

White, R. L., Becker, R. H., \& Helfand, D. J. 2005, AJ, 130, 586 
Williams, J. P., Blitz, L., \& McKee, C. F. 2000, Protostars and

Planets IV, 97 